\title{
Nasal Rhinosporidiosis in South Africa: Review of Literature and Report of a Case.
}

SADJ October 2017, Vol 72 no 9 p420 - p423

MMJ Masilela', MS Selepe ${ }^{2}$, L Masilo. ${ }^{3}$

\begin{abstract}
Background: Rhinosporidiosis is a rare chronic granulomatous infection presenting primarily on the mucous membrane of nasal cavities, nasopharynx and oral cavity. Rhinosporidium seeberi has been identified as the causative agent; however recent studies have implicated a waterborne organism, the cyanobacterium Microcystis aeruginosa, as the cause. It is endemic in India and Sri Lanka where $90 \%$ of all infections occur. The aim of this paper is to review literature on rhinosporidiosis and to report on one of the sporadic cases encountered in South Africa, Gauteng province.
\end{abstract}

Case presentation: A 17 year old black male patient presented with a pedunculated nasal mass causing nasal obstruction. Microscopical examination showed a polypoid mass with numerous thick walled sporangia in a background of fibrovascular connective tissue stroma with granulomatous inflammation. Fluorescent microscopy was effective in identifying the walls of the sporangia in the granulomas. A final diagnosis of nasal rhinosporidiosis was made.

Conclusion: Rhinosporidiosis, a rare condition in our environment, has the potential to disseminate to other parts of the body. It is therefore critical for the clinicians to always consider rhinosporidiosis as a differential diagnosis when assessing patients presenting with nasal polyps.

Keywords: Nasal polyp; Rhinosporidium seeberi; Sporangia with endospores; Microcystis aeruginosa; Rhinosporidiosis in South Africa.

1. Mokgobja MJ Masilela: Department of Oral Pathology, Schoo of Oral Health Science, Sefako Makgatho Health Sciences University, South Africa.

2. Manoko S Selepe: Department of Otorhinolaryngology, Schoo of Medicine, Sefako Makgatho Health Sciences University, South Africa.

3. Letlhogonolo Masilo: Department of Oral Pathology, School of Oral Health Science, Sefako Makgatho Health Sciences University, South Africa.

Corresponding author

Mokgobja MJ Masilela:

Department of Oral Pathology, School of Oral Health Science, Sefako Makgatho Health Sciences University, South Africa.

Email:Mokgobja.Masilela@smu.ac.za

\section{BACKGROUND}

Rhinosporodiosis is a rare chronic granulomatous infection that presents as sessile or pedunculated polypoid lesion, primarily affecting the mucous membrane of nasal cavities, nasopharynx and oral cavity. ${ }^{1,2}$ Whilst extranasal involvement is rare, skin, eyes and bone, amongst other sites, may also be affected and it can occasionally present as disseminated disease..$^{3-5}$ The condition is believed to be caused by Rhinosporidium seeberi but recent studies have implicated a waterborne organism, the cyanobacterium Microcystis aeruginosa. ${ }^{1,6,7}$ A molecular study on the polyps of rhinosporidiosis detected 16S rRNA gene in round bodies. ${ }^{6}$ This confirmed Microcystis aeruginosa as a causative agent. The presumed mode of infection is through traumatised epithelium, most commonly in nasal sites. The spread could be autoinoculation, or haematogenous or lymphatic spread. ${ }^{7}$ It may occur anywhere but is endemic in India and Sri Lanka where $90 \%$ of all infections are recorded. ${ }^{1}$

Microscopically, rhinosporidiosis is characterized by hyperplastic respiratory or squamous epithelium, accompanied by prominent subepithelial chronic granulomatous inflammation. The connective tissue stroma contains numerous thick walled, 100 to 300 microns sporangia that are in different stages of maturity. Within the sporangia are numerous endospores with a characteristic arrangement of immature and mature forms. ${ }^{1}$ The condition is rare in African continent and to date only 14 cases have been reported from South Africa. ${ }^{4,8,9}$

\section{CASE REPORT}

A 17 year old male patient presented with a pedunculated polypoid mass attached to the anterior part of the nasal septum, causing nasal obstruction. The lesion was clinically suspected to be an allergic polyp. Excisional biopsy was done and the specimen was submitted for histological examination. Microscopy revealed a polypoid mass covered by respiratory epithelium with areas of squamous metaplasia. The core of the lesion showed numerous thick walled sporangia in different stages of maturity within the background of a fibrovascular connective tissue stroma with dense granulomatous inflammation (Figure 1). Evidence of fusion of some sporangia between the overlying epithelium and ductal lining, with a resultant rupture and release of the endospores onto the overlying surface epithelium and into 

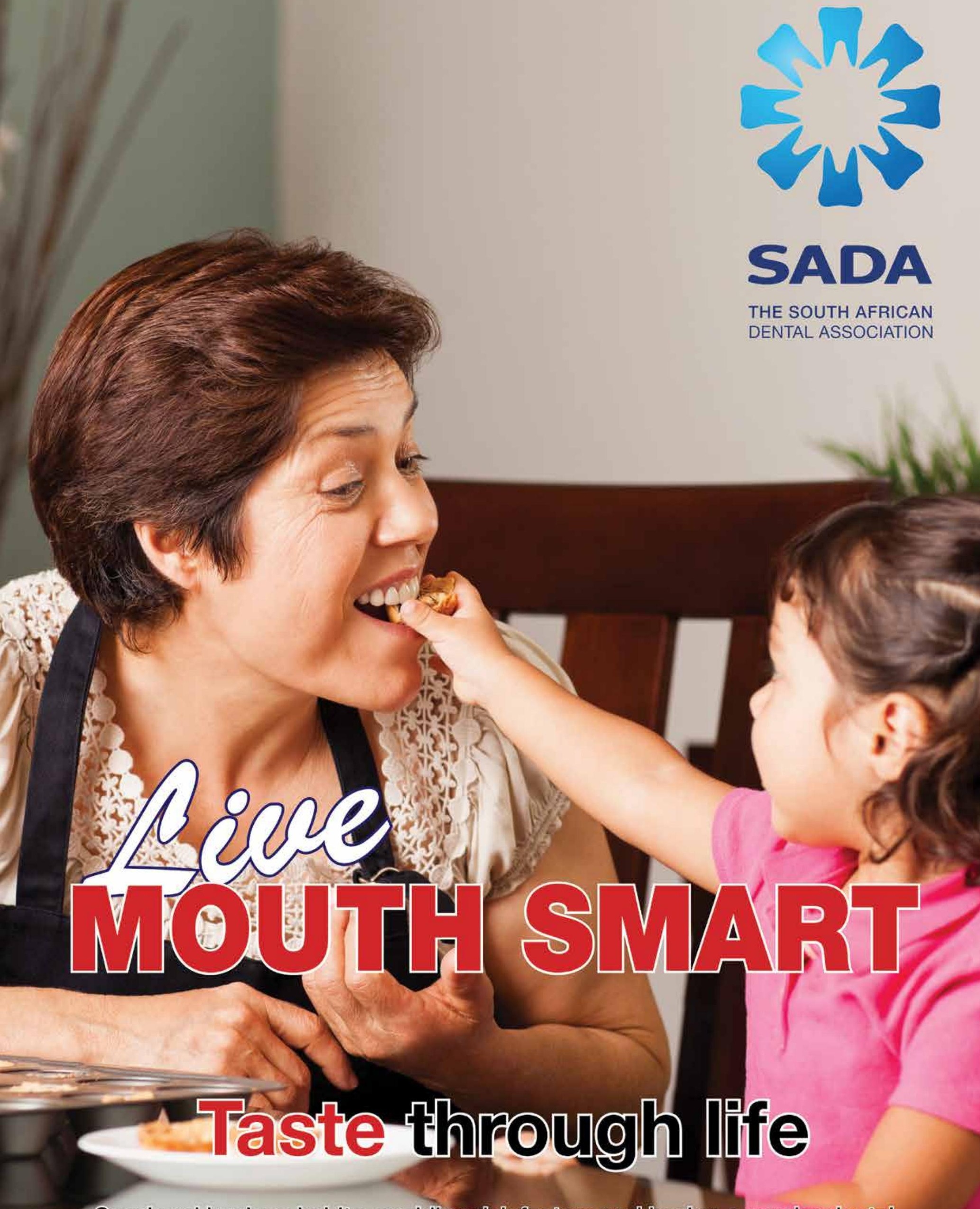

THE SOUTH AFRICAN DENTAL ASSOCIATION

Good oral hygiene habits, avoiding risk factors and having a regular dental check-up from early in life cann help maintain optinnal oral health into old age.

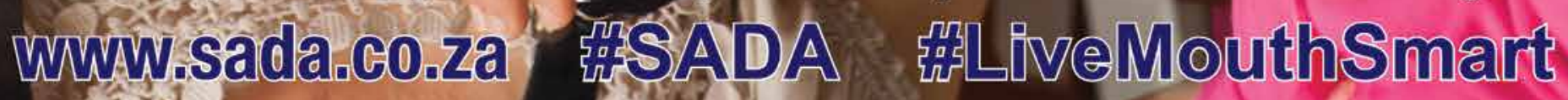


the ductal lumen respectively, was also demonstrated. There were foci of foreign body giant cell reactions that were probably a reaction to the endospores released from ruptured sporangia. Mucicarmine stained the inner walls of the capsules of the sporangia and the endospores (Figure 2). The fluorescent microscope demonstrated autofluorescence of the capsules of the sporangia and endospores (Figure 3).

\section{DISCUSSION}

Rhinosporodiosis is a rare condition in the African continent with only a few sporadic cases having been reported in the literature. The condition has the highest incidence in India and Sri Lanka. ${ }^{1}$ Although to date only 14 cases have been recorded from South Africa ${ }^{4,8,9}$ others have been reported in Burundi, Zaire, Rwanda, Tanzania, Chad, Malawi, Kenya, Uganda, Congo and Egypt.10-13 Almost all the South African cases were ocular rhinosporidiosis and were encountered in patients younger than 15 years of age. This is the first case to be reported in Gauteng province and it is also that of a younger patient. The case was diagnosed in the Department of Oral Pathology of Medunsa Oral Health Centre, Sefako Makgatho Health Sciences University. The biopsy was submitted by the Department of Otorhinolaryngology, Dr George Mukhari Academic Hospital.

It is believed that stagnant ground water is the natural habitat of the causative agent. Accordingly it will be important to determine the chemical and physical characteristics of those waters where it is likely that these South African individuals were infected. Host predisposing factors such as blood group and genotype HLA types, should also be investigated as there could be many people who are bathing in ponds and lakes; yet the incidence of this disease is still low in the country. The incidence of rhinosporidiosis has been reported to be the highest in individuals of blood Group $O$ followed by Group AB. ${ }^{7}$ Recurrences and dissemination of the disease should also be investigated and documented.

The clinical presentation of rhinosporidiosis can mimic other non-infectious nasal polyps. It is therefore critical to take a biopsy for histological examination of the lesion to confirm a definitive diagnosis. The organisms can be identified easily on sections stained by hematoxylin and eosin. Other histochemical stains such as Periodic Acid Schiff and mucicarmine can also be used to establish the correct diagnosis of rhinosporidiosis. The autofluorescence demonstrated by the capsules of the sporangia and endospores revealed distinctly the capsule of a ruptured sporangium with release of endospores and resultant foreign body giant cell reaction. The property of autofluorescence was also observed by other authors. ${ }^{14}$

It is important that the incisional or excisional biopsy is taken with caution to avoid spillage of endospores from the polyp. Spillage is usually followed by autoinoculation through the adjacent epithelium and this tends to serve as a base for increased recurrences in rhinosporidiosis. ${ }^{15}$ Hence the recommended treatment is a combination of surgical excision and electrocoagulation. ${ }^{1}$

Just as the clinical presentation can mimic certain conditions so may to the histological features and the following differential diagnoses need to be considered: Coccidioides immitis, oncocytic Schneiderian papilloma and Myospherulosis.

Coccidioides immitis also evoke a granulomatous foreign body giant cell reaction with a dense chronic inflammatory infiltrate. However; the spherules of Coccidioides immitis measure approximately 30 - $80 \mu$ as compared with those of Rhinosporidiosis seeberi which measure $100-350 \mu$. The endospores are small and numerous measuring $2-5 \mu$ whereas those of Rhinosporidiosis seeberi measure 5-10 $\mu$. The capsule of Rhinosporidiosis seeberi has a thicker, distinctly bilaminated capsule that stains with mucicarmine whereas the capsule of Coccidioides immitis does not. Also this type of infection is not common in the nasal cavity. ${ }^{16}$

In oncocytic Schneiderian papilloma, the epithelial lining has scattered mucous cells with intraepithelial microcysts that contain mucin and polymorphonuclear cells. The surface epithelium in rhinosporidiosis lacks oncocytes and there is no endophytic growth. The microcysts do not have a thick capsule and there are no contained endospores. ${ }^{17}$

Myolospherulosis is a rare iatrogenic pseudomycotic lesion characterized by prominent fibrous and chronic inflammation with foreign body-type giant cells surrounding pseudocystic spaces. The spaces contain saclike structures with a thick dark wall, referred to as parent bodies with enclosed "fungus-like" spherules. These spherules are simply red blood cells that have been altered by the medication. This is actually a result of a tissue interaction 
with petroleum base and an antibiotic.,18 The spherules do not stain with mucicarmine.

Surgical resection is the primary treatment for rhinosporidiosis and this may be combined with electrocoagulation. ${ }^{1}$ If the lesions are not treated early, dissemination to distant areas, usually attributed to haematogenous spread, may occur. There has been a report of a case where a single patient had simultaneous involvement of the skin, subcutaneous tissue, muscle, bone, penis, urethra and nasal cavity. ${ }^{19}$ One case was reported of a patient with nasal rhinosporidiosis with dissemination to a finger, necessitating amputation. ${ }^{5}$

\section{CONCLUSION}

Rhinosporidiosis is a rare condition in our region. The disease has the potential to disseminate to other parts of the body. Clinicians should always consider rhinosporidiosis as a differential diagnosis when assessing patients presenting with nasal polyps.

Recommendation: It is important for all health care institutions in Africa to report this rare disease, so that definitive reference data can be accumulated on the geographic and demographic distribution of rhinosporidiosis. Further investigations should be undertaken to establish those host factors that render patients susceptible to this infection.

Consent: Written informed consent was obtained from the patient for publication of this Case report and any accompanying images.

Ethics: Approval to report the case was obtained the rom the Research Ethics Committee of the Sefako Makgatho Health Sciences University The reference number is SMUREC/D/160/2015: IR

\section{Conflict of interest: None declared}

\section{References}

1. Prasad ML, Perez-Ordonez. Nonsquamous lesions of the nasal cavity, paranasal sinuses, and nasopharynx. in: Gnepp DR, ed. Diagnostic Surgical Pathology of the Head and Neck. Philadelphia: Saunders, 2009: 117.

2. Morelli L, Polce M, Piscioli Fet al. Human nasal rhinosporidiosis: An Italian case report. Diagnostic Pathology 2006; 1: 25.

3. Prasad K, Veena S, Permi HS, Teerthanath S, Shetty KP, Shetty JP. Disseminated cutaneous rhinosporidiosis. Journal of Laboratory Physicians 2010; 2(1): 44-6.

4. Salazar Campos MC, Surka J, Garcia Jardon M, Bustamante N. Ocular rhinosporidiosis. South African Medical Journal 2005; 95: 950-2.

5. Kundu AK, Puljhele S, Jain M, Srivastava RK. Osseous involvement in rhinosporidiosis. Indian J Orthop 2013; 47: 523-5.

6. Dhaulakhandi DB, Ahluwalia KB, Ravi AK, Gang LC. Detection of $16 \mathrm{~S}$ rRNA gene in round bodies isolated from polyps of rhinosporidiosis. Infection, Genetica and Evolution 2006; 6(4): 331-6.

7. Arseculeratne SN. Recent advances in rhinosporidiosis and rhinosporidium seeberi. Indian J Med Microbiol 2002; 20: 119-31.

8. Chetty R, Cooper K. Rhinosporidiosis at King Edward VIII Hospital, Durban - 1976 - 1985. A report of six cases. S Afr Med J 1987; 72: 217-8.

9. Smith PL. Rhinosporidiosis: case reports. S Afr Med J 1977; 51: 281.

10. Gigase P, Kestelyn P. Further African cases of rhinosporidiosis. Ann.Soc.Belge Med Trop 1993; 73: 149-52.

11. Uledi S, Fauzia A. Human nasal rhinosporidiosis: a case report from Malawi. Pan Afr Med J 2011; 9: 27.
12. Ondzotto G. Intranasal rhinosporidiosis: presentation of the first case seen in the Congo. Bull Soc Pathol Exot 2002; 95(2): 78-80.

13. Hussein MR, Rashad UM. Rhinosporidiosis in Egypt: a case report and review of literature. Mycopathologia 2005; 159(2): 205-7.

14. Moses JS, Balachandran C, Singh B, Shanmugan A, Moses D. Rhinosporidium seeberi: Light, phase contrast, fluorescent and scanning electron microscopic study. Mycopathologia 1991; 114: 17-20.

15. Kurunaratne WAE. Rhinosporidiosis in Man: The Athlone Press, London, 1964.

16. Brandwein-Gensler MS, Mahadevia P, Gnepp DR. Nonsquamous pathologic diseases of the hypopharynx, larynx, and trachea. In: Gnepp DR, ed. Diagnostic Surgical Pathology of the Head and Neck. Philadelphia: Saunders, 2009: 319-20.

17. Neville BW, Damm DD, Allen CM, Chi AC. Oral and Maxillofacial Pathology. 4th ed. St. Louis: Elsevier, 2016: 340.

18. Neville BW, Damm DD, Allen CM, Chi AC. Oral and Maxillofacial Pathology. 4th ed. St. Louis: Elsevier, 2016: 297-8.

19. Bandyopadhyay SN, Das S, Majhi TK, Bandyopadhyay G, Roy $D$. Disseminated rhinosporidiosis. The Journal of Laryngology and Otology 2013; 127: 1020-2. 